\title{
Clinical characteristics and long-term outcomes for patients who undergo cytoreductive surgery for thoracic meningiomas: a retrospective analysis
}

\author{
Leonel Ampie, MD, ${ }^{1,2}$ M. Harrison Snyder, BS, ${ }^{1}$ Jose F. Dominguez, MD, ${ }^{3}$ Avery Buchholz, MD, ${ }^{1}$ \\ Chun-Po Yen, MD, ${ }^{1}$ Mark E. Shaffrey, MD, ${ }^{1}$ Hasan R. Syed, MD, ${ }^{1}$ Christopher I. Shaffrey, MD, ${ }^{4}$ and \\ Justin S. Smith, MD, PhD'
}

${ }^{1}$ Department of Neurological Surgery, University of Virginia Health System, Charlottesville, Virginia; ${ }^{2}$ Surgical Neurology Branch, National Institute of Neurological Disorders and Stroke, National Institutes of Health, Bethesda, Maryland; '3epartment of Neurosurgery, Westchester Medical Center, New York Medical College, Valhalla, New York; and ${ }^{4}$ Department of Neurological Surgery, Duke University, Durham, North Carolina

\begin{abstract}
OBJECTIVE Primary spinal meningiomas represent a rare indolent neoplasm usually situated in the intradural-extramedullary compartment. They have a predilection for afflicting the thoracic spine and most frequently present with sensory and/or motor symptoms. Resection is the first-line treatment for symptomatic tumors, whereas other clinical factors will determine the need for adjuvant therapy. In this study, the authors aimed to elucidate clinical presentation, functional outcomes, and long-term outcomes in this population in order to better equip clinicians with the tools to counsel their patients. METHODS This is a retrospective analysis of patients treated at the authors' institution between 1998 and 2018. All patients with thoracic meningiomas who underwent resection and completed at least one follow-up appointment were included. Multiple preoperative clinical variables, hospitalization details, and long-term outcomes were collected for the cohort.

RESULTS Forty-six patients who underwent resection for thoracic meningiomas were included. The average age of the cohort was 59 years, and the median follow-up was 53 months. Persistent sensory and motor symptoms were present in 29 patients (63\%). Fifteen lesions were ventrally positioned. There were $43 \mathrm{WHO}$ grade I tumors, 2 WHO grade II tumors, and $1 \mathrm{WHO}$ grade III tumor; the grade III tumor was the only case of recurrence. The median length of hospitalization was 4 days. Seventeen patients (37\%) were discharged to rehabilitation facilities. Thirty patients $(65.2 \%)$ experienced resolution or improvement of symptoms, and there were no deaths within 30 days of surgery. Only 1 patient developed painful kyphosis and was managed medically. Ventral tumor position, new postoperative deficits, and length of stay did not correlate with disposition to a facility. Age, ventral position, blood loss, and increasing WHO grade did not correlate with length of stay.
\end{abstract}

CONCLUSIONS Outcomes are overall favorable for patients who undergo resection of thoracic meningiomas. Symptomatic patients often experience improvement, and patients generally do not require significant future operations. Tumors located ventrally, while anatomically challenging, do not necessarily herald a significantly worse prognosis or limit the extent of resection.

https://thejns.org/doi/abs/10.3171/2021.2.FOCUS20977

KEYWORDS spine; spinal cord neoplasms; benign; intradural extramedullary; meningioma; patient outcome assessments

$\mathrm{P}$ ATIENTS harboring primary spinal neoplasms represent a rare cohort. Review of a national database found that meningiomas $(24.4 \%)$ are the most common of these tumors, followed by ependymomas (23.7\%), and schwannomas (21.2\%) in a cohort of 430 patients with primary intraspinal tumors. ${ }^{1,2}$ Meningiomas, in particular, account for the most common primary spinal intradural tumor. Since female patients have a greater incidence of developing both intracranial and spinal meningiomas, sex hormones may play a role in tumor development and growth. ${ }^{1,2}$ Treatment modalities for meningiomas are relegated to operative cytoreduction and radiation therapy, depending on the extent of resection as well as WHO tumor grade, with more aggressive tumors often requiring adju-

ABBREVIATIONS ASA = American Society of Anesthesiologists; EBL = estimated blood loss; GTR = gross-total resection; LOS = length of stay; NF2 = neurofibromatosis type 2; PMHx = past medical history; STR = subtotal resection.

SUBMITTED December 17, 2020. ACCEPTED February 16, 2021.

INCLUDE WHEN CITING DOI: 10.3171/2021.2.FOCUS20977. 
vant therapy. Deciding whether or not to pursue aggressive tumor resection depends on patient symptoms, age, and other clinical factors, as these neoplasms tend to have an indolent growth pattern.

Most primary spinal meningiomas tend to be benign WHO grade I lesions; WHO grade II and III tumors are rarely encountered. Presenting symptoms depend on location and size, but most patients typically present with chronic back pain with subsequent neuraxis imaging demonstrating the lesion. Other common presenting symptoms include sensory disturbance and bowel/bladder dysfunction for lesions in the lumbosacral/cauda equina region. Approximately $55 \%-83 \%$ of spinal meningiomas arise in the thoracic spinal cord, followed by $15 \%$ in the cervical spine, and $2 \%$ in the lumbar spine..$^{2-7}$ Multilevel decompression may be required to develop an appropriate operative corridor, but this can lead to spinal instability over time. Some older patients may also be at higher risk of instability if they have poor bone density quality. The thoracic spine is usually protected from similar instability in the face of multilevel bony decompression as opposed to the cervical and lumbar spine due to added support from adjacent osseous structures. In this study, we focused on thoracic spinal cord lesions specifically, as their incidence is higher in this region, and we assessed preoperative symptom profile; intraoperative complications, including changes in neuromonitoring; recurrence rate; and long-term clinical follow-up.

\section{Methods}

This retrospective study was approved by the University of Virginia (UVA) institutional review board. A list of all central nervous system meningiomas treated at UVA between the years 1998 and 2018 were compiled from the billing department. From a database consisting of patients with benign intracranial and spinal tumors, there were 101 spinal tumors operated on at UVA, of which 74 were meningiomas; 22 occurred in the cervical spine, 50 in the thoracic spine, and 2 in the lumbar spine. We excluded patients with any nonmeningioma tumor, a tumor outside the thoracic spine, and any patient who had no available clinical follow-up information. We extracted a total of 46 patients who had meningiomas specifically in the thoracic spine and who had available clinical follow-up. We collected the following variables: age, sex, preoperative motor and sensory symptoms, past medical history (PMHx), size, American Society of Anesthesiologists (ASA) physical status score, use of preoperative steroids, number of levels that underwent laminectomies, concurrent or future need for spinal instrumentation, estimated blood loss (EBL), intraoperative fluid administration, urine output, neuromonitoring changes, Simpson grade, postoperative complications, WHO grade, pathological subtype, length of follow-up, adjuvant therapy, recurrence, and death. Follow-up generally entailed a clinic visit within 2-3 weeks of surgery and at $2-3$ months, followed by yearly visits unless ongoing treatment was needed. Postoperative imaging was performed 2-3 months after discharge and then yearly thereafter.

\section{Statistical Analysis \\ SPSS Statistics version 26 (IBM Corp.) was used for}

logistic regression. A binary logistic regression model was used to analyze disposition to rehabilitation facilities, and linear regression was used to analyze factors associated with increasing length of stay (LOS).

\section{Results \\ Patient Demographics}

A total of 46 patients with a thoracic spinal cord meningioma were identified (Table 1). The majority of the patients were female, making up $80.4 \%$ of the cohort ( $\mathrm{n}=$ 37). The mean age was $59 \pm 18$ years (range $17-90$ years). The median clinical follow-up was 53 months (range 1-201 months), and the median radiographic follow-up was 27 months (range 1-180 months). Thirty-four patients (73.9\%) had follow-up of at least 2 years. This was the first spinal tumor diagnosed in the majority of patients $(91.3 \%$, $\mathrm{n}=42$ ). The patients were generally healthy, with the majority having a PMHx of hypertension (34.8\%), diabetes (17.4\%), or any previously diagnosed neoplasm (26.1\%; 2 breast cancer, 1 angiomyolipoma, 3 neurofibromatosis, 2 previous spinal meningiomas, 1 von Hippel-Lindau syndrome, 1 nonmelanoma skin cancer, 1 gastrointestinal stromal tumor, and 1 with lung cancer and melanoma). Three patients had a genetic diagnosis of neurofibromatosis type 2 (NF2) (6.5\%). None of the patients with diagnosed NF2 had recurrence at the time of the last followup. The anesthesiology team considered most patients to be safe for surgery, as $63 \%$ of patients were in ASA class II. The most common presenting symptoms were persistent sensory disturbance (63\%) and either subjective or objective motor weakness (63\%). The majority of the meningiomas were allocated to the upper thoracic region between $\mathrm{T} 1$ and $\mathrm{T} 4$ (45.7\%), followed by the midthoracic T5-8 region (28.3\%), and lower thoracic region T9-12 (26.1\%). All patients had intradural extramedullary lesions except 1 patient who had an infiltrative intramedullary lesion that was confirmed to be an anaplastic meningioma. Lesions were mostly dorsally located $(65.2 \%$, $\mathrm{n}=31$ ) as opposed to being ventral to the spinal cord $(32.6 \%, \mathrm{n}=15)$; there was only 1 patient whose lesion was unspecified as we did not have the preoperative imaging available for review.

\section{Operative and Postoperative Details}

Surgical details are summarized in Table 2. The patients generally required a 2 -level $(45.7 \%$, mean tumor diameter $18.1 \mathrm{~mm}, 66.7 \%$ dorsal) or a 3-level $(43.5 \%$, mean tumor diameter $18.7 \mathrm{~mm}, 68.4 \%$ dorsal) decompression to obtain sufficient access to the meningioma. Two patients $(4.3 \%)$ required a 4-level laminectomy (mean tumor diameter $26 \mathrm{~mm}, 50 \%$ dorsal) and 1 patient $(2.2 \%)$ required a 5-level laminectomy (tumor diameter $16 \mathrm{~mm}$, dorsal). One patient underwent concurrent instrumentation at the time of resection for a tumor near the thoracolumbar region located at T11-12. Twenty patients (43.5\%) underwent Simpson grade 2 resections (mean tumor diameter $17.1 \mathrm{~mm}, 55 \%$ dorsal) followed by $28.3 \%$ who underwent Simpson grade 3 resections (mean tumor diameter 18.5 $\mathrm{mm}, 84.6 \%$ dorsal), $17.4 \%$ underwent Simpson grade 4 resections (mean tumor diameter $22 \mathrm{~mm}, 62.5 \%$ dorsal), 
TABLE 1. Baseline characteristics

\begin{tabular}{|c|c|}
\hline Variable & Value \\
\hline Mean age, yrs & $59 \pm 18(17-90)$ \\
\hline Female sex, $\mathrm{n}(\%)$ & $37(80.4)$ \\
\hline First spinal tumor, n (\%) & $42(91.3)$ \\
\hline \multicolumn{2}{|l|}{ PMHx, n (\%) } \\
\hline Hypertension & $16(34.8)$ \\
\hline Diabetes mellitus & $8(17.4)$ \\
\hline Past tumors (any type) & $12(23.9)$ \\
\hline NF2 & $3(6.5)$ \\
\hline Lung disease & $5(10.9)$ \\
\hline \multicolumn{2}{|l|}{ Presenting symptoms, n (\%) } \\
\hline Transient sensory & $12(23.9)$ \\
\hline Persistent sensory & $29(63)$ \\
\hline Motor & $29(63)$ \\
\hline No symptoms & $2(4.3)$ \\
\hline Preop steroids given, $\mathrm{n}(\%)$ & $6(13)$ \\
\hline \multicolumn{2}{|l|}{ Tumor location, $\mathrm{n}(\%)$} \\
\hline Upper thoracic (T1-4) & $21(45.7)$ \\
\hline Midthoracic (T5-8) & $13(28.3)$ \\
\hline Lower thoracic (T9-12) & $12(26.1)$ \\
\hline \multicolumn{2}{|l|}{ Dural relation, $\mathrm{n}(\%)$} \\
\hline Intradural, extramedullary & $45(97.8)$ \\
\hline Intradural, intramedullary & $1(2.2)$ \\
\hline \multicolumn{2}{|l|}{ Position w/in spinal canal, $\mathrm{n}(\%)$} \\
\hline Dorsal & $31(65.2)$ \\
\hline Ventral & $15(32.6)$ \\
\hline \multicolumn{2}{|l|}{ Enhancement, n (\%) } \\
\hline Homogeneous & $25(54.3)$ \\
\hline Heterogeneous & $9(19.6)$ \\
\hline Unclear & $5(10.9)$ \\
\hline Preop MRI not available & $7(15.2)$ \\
\hline Mean largest tumor diameter, $\mathrm{mm}^{*}$ & $18.4 \pm 6.7(9-43)$ \\
\hline \multicolumn{2}{|l|}{ ASA grade, $n(\%)$} \\
\hline 1 & $1(2.2)$ \\
\hline II & $29(63)$ \\
\hline III & $16(34.8)$ \\
\hline Median total follow-up, mos & $53(1-201)$ \\
\hline
\end{tabular}

Mean values are presented as the mean \pm SD (range).

* Reported for 39 lesions.

and only $10.9 \%$ underwent Simpson grade 1 resections (mean tumor diameter $20.3 \mathrm{~mm}, 80 \%$ dorsal). The mean blood loss was not substantial, with a mean EBL of $228 \pm$ $126 \mathrm{ml}$ (range 50-600 ml). The mean intraoperative fluid administration was $2336 \pm 913 \mathrm{ml}$ (range 1000-6000 $\mathrm{ml}$ ), and the mean urine output was $663 \pm 665 \mathrm{ml}$ (range 100-3300 ml). Postoperative medical complications were minimal, with 1 patient who had a pulmonary embolism, 1 patient with a hemorrhagic infarct during surgery, and 1 patient who required a repeat surgery to repair a CSF leak. About $10.9 \%$ of patients did have a new postoperative neurological deficit. Notably, 3 patients had any type
TABLE 2. Surgical course

\begin{tabular}{|c|c|}
\hline Variable & Value \\
\hline \multicolumn{2}{|l|}{ No. of laminectomy levels, $n(\%)$} \\
\hline 1 & $2(4.3)$ \\
\hline 2 & $21(45.7)$ \\
\hline 3 & $20(43.5)$ \\
\hline 4 & $2(4.3)$ \\
\hline 5 & $1(2.2)$ \\
\hline Instrumentation, $\mathrm{n}(\%)$ & $1(2.2)$ \\
\hline \multicolumn{2}{|l|}{ Extent of resection, $\mathrm{n}(\%)$} \\
\hline GTR & $38(82.6)$ \\
\hline STR & $8(17.4)$ \\
\hline \multicolumn{2}{|l|}{ Simpson grade of resection, $n(\%)$} \\
\hline 1 & $5(10.9)$ \\
\hline 2 & $20(43.5)$ \\
\hline 3 & $13(28.3)$ \\
\hline 4 & $8(17.4)$ \\
\hline \multicolumn{2}{|l|}{ Neuromonitoring disruptions, $\mathrm{n}(\%)$} \\
\hline None & $34(73.9)$ \\
\hline Mild & $2(4.3)$ \\
\hline Severe & $1(2.2)$ \\
\hline Not used & $9(19.6)$ \\
\hline Mean EBL, ml & $228 \pm 126(50-600)$ \\
\hline Mean intravenous fluid, ml & $2336 \pm 913(1000-6000)$ \\
\hline Mean urine output, ml & $663 \pm 665(100-3300)$ \\
\hline \multicolumn{2}{|l|}{ WHO grade, $n(\%)$} \\
\hline I & $43(93.5)$ \\
\hline II & $2(4.3)$ \\
\hline III & $1(2.2)$ \\
\hline \multicolumn{2}{|l|}{ Pathological features, $\mathrm{n}(\%)$} \\
\hline Necrosis & $2(4.3)$ \\
\hline Hypercellularity & $1(2.2)$ \\
\hline \multicolumn{2}{|l|}{ Postop/hospital complications, $\mathrm{n}(\%)$} \\
\hline Altered mental status & $1(2.2)$ \\
\hline Anemia requiring transfusion & $1(2.2)$ \\
\hline CSF leak requiring operative repair & $1(2.2)$ \\
\hline $\begin{array}{l}\text { Hematomyelia \& hemorrhagic infarct } \\
\text { requiring surgery }\end{array}$ & $1(2.2)$ \\
\hline Pulmonary embolism & $1(2.2)$ \\
\hline Perioperative mortality & $0(0)$ \\
\hline New postop neurologic deficit, n (\%) & $5(10.9)$ \\
\hline Median LOS, days (range) & $4(1-9)$ \\
\hline \multicolumn{2}{|l|}{ Disposition, $\mathrm{n}(\%)$} \\
\hline Home & $29(63)$ \\
\hline Rehab & $17(37)$ \\
\hline Adjuvant RT, n (\%) & $2(4.3)$ \\
\hline
\end{tabular}

$\mathrm{RT}=$ radiotherapy.

Mean values are presented as the mean \pm SD (range). 
TABLE 3. Outcomes

\begin{tabular}{lc}
\hline \multicolumn{1}{c}{ Variable } & Value \\
\hline \multicolumn{2}{c}{ Status of symptoms at last follow-up, $\mathrm{n}(\%)$} \\
\hline Resolved & $16(34.8)$ \\
\hline Improved, but still present & $14(30.4)$ \\
\hline Stable & $9(19.6)$ \\
\hline Worsened or new & $6(13)$ \\
\hline No further objective physical exam data & $1(2.2)$ \\
\hline Recurrence, $\mathrm{n}(\%)$ & $1(2.2)$ \\
\hline Repeat surgery, $\mathrm{n}(\%)$ & $1(2.2)$ \\
\hline Other treatment after recurrence, $\mathrm{n}(\%)$ & $1(2.2)$ \\
\hline RT & $1(2.2)$ \\
\hline Medical treatment & $5(10.9)$ \\
\hline Death during follow-up, $\mathrm{n}(\%)$ & $0(0)$ \\
\hline Death w/in 30 days of surgery, $\mathrm{n}(\%)$ & $68.4 \pm 15.7(51-86)$ \\
\hline Mean age at death, yrs &
\end{tabular}

Mean values are presented as the mean \pm SD (range).

of neuromonitoring disruption that was noted intraoperatively ( 2 mild, 1 severe), and only the patient with a severe disruption developed postoperative deficits. The average LOS in the hospital was approximately 4 days (range 1-9 days) and most patients were discharged to home $(\mathrm{n}=$ $29,63 \%)$ as opposed to a rehabilitation facility $(\mathrm{n}=17$, $36.9 \%$ ). Two patients required adjuvant radiotherapy (1 with a WHO grade III anaplastic meningioma, and 1 with a WHO grade I tumor that was subtotally resected). Thirty patients had improvement or resolution of symptoms, while 6 had worsening or new symptoms postoperatively. Outcomes are summarized in Table 3.

\section{Neuropathology}

All lesions were sent for both frozen and permanent pathology; they were reviewed by a trained neuropathologist. The majority of patients $(93.5 \%)$ had a WHO grade I meningioma while $4.3 \%$ had a WHO grade II meningioma, and only 1 patient had an anaplastic WHO grade III meningioma. The majority of patients had psammomatous subtype meningiomas $(\mathrm{n}=11)$, followed by meningothelial $(\mathrm{n}=8)$, multiple subtypes $(\mathrm{n}=3)$, metaplastic ( $=2)$, atypical $(\mathrm{n}=2)$, transitional $(\mathrm{n}=1)$, and otherwise unspecified $(\mathrm{n}=18)$.

\section{Discharge and LOS}

Binomial logistic regression was used to analyze factors associated with increased risk of discharge to rehabilitation facilities (Table 4). None of the factors among age at surgery, ventral spinal canal position, new postoperative deficit, and LOS achieved significance, although increasing age trended toward significance $(\mathrm{p}=0.059)$. The average age of patients discharged to a rehabilitation facility was 67.4 years, and the average age for those discharged to home was 55.2 years. Factors predicting LOS were analyzed using linear regression (Table 4); age, ventral position, EBL, and increasing WHO grade were not significant.
TABLE 4. Regression analysis for disposition to rehabilitation facilities (binomial) and LOS (linear)

\begin{tabular}{llll}
\hline \multicolumn{1}{c}{ Variable } & $p$ Value & OR & $95 \% \mathrm{Cl}$ \\
\hline Discharge to rehab facility & & & \\
\hline Age at surgery & 0.059 & 1.045 & 0.998 to 1.94 \\
\hline Ventral position in canal & 0.964 & 0.968 & 0.236 to 3.97 \\
\hline New postop deficit & 0.558 & 1.85 & 0.236 to 14.504 \\
\hline LOS & 0.311 & 1.182 & 0.855 to 1.634 \\
\hline LOS & & & \\
\hline Age at surgery & 0.555 & & -0.051 to 0.028 \\
\hline Ventral position in canal & 0.773 & & -1.717 to 1.285 \\
\hline EBL & 0.299 & & -0.009 to 0.003 \\
\hline Increasing WHO grade & 0.346 & & -2.349 to 0.844 \\
\hline
\end{tabular}

\section{Discussion}

Patients with spinal cord neoplasms, specifically meningiomas, represent a rare cohort, as primary neoplasms arising from the spinal axis are uncommon. Meningiomas represent the most common primary spinal neoplasm, and they have a predilection for the thoracic spine. ${ }^{8-10}$ Most tend to represent WHO grade I neoplasms, with low mitotic activity and low risk of recurrence. These tumors also engender a very low annual growth rate, and resection is only considered depending on preoperative symptoms or if the tumor is large enough that it would necessitate intervention to prevent spinal cord compression. Nonetheless, it is important to describe the demographic information, long-term postoperative outcome, and recurrence rate for these patients.

\section{Preoperative Presentation}

Most patients presented during their 5th or 6th decade of life and were generally healthy, with expected PMHx of hypertension and diabetes. They also had low operative morbidity scores per the ASA classification. The most common presenting symptoms included permanent sensory and subjective/objective weakness; 2 patients had asymptomatic lesions. These findings are similar to those reported by Yamaguchi et al. in a retrospective study demonstrating that motor weakness and sensory disturbance were more common in patients with thoracic spine meningiomas than in the cervical or lumbar spine (no cases of the latter were reported in the study). ${ }^{2}$

\section{Operative Details and Patient Disposition}

Twenty-five patients had gross-total resection (GTR; Simpson grade 1 or 2) and 21 had a subtotal resection (STR; Simpson grade 3 or 4). Notably, all patients underwent standard midline incisions and laminectomies. None of the patients required parascapular extrapleural, extracavitary, or retropleural approaches. In this cohort, lesions treated with Simpson grade 3 or 4 resections had average sizes of $18.5 \mathrm{~mm}$ and $22 \mathrm{~mm}$, respectively, while lesions treated with grade 1 or 2 resections had an average maximum diameter of $20.3 \mathrm{~mm}$ and $17.1 \mathrm{~mm}$, respectively. Resection grade and ventral position within the canal did 
not impact recurrence rate in this cohort, as only 1 tumor recurred in a patient with high-grade meningioma. Intraoperative neuromonitoring was performed in 37 patients, with only 3 displaying any changes. One of these patients had significant disruption of motor potentials of the right lower extremity and demonstrated weakness in the affected limb, which resolved. In general, the procedures were well tolerated with only mild, transient deficits, if any occurred. Ventrally located meningiomas represent a challenge and require an approach specifically tailored to the particular anatomy of each case. ${ }^{11-13}$ In this cohort, 15 ventral meningiomas were treated, and GTR was achieved in 10 cases. Imaging examples in patients with dorsally and ventrally located tumors are shown in Fig. 1. None of these patients experienced worsened symptoms at the last follow-up (11 resolved or improved). Although anatomically challenging, surgery for ventral lesions appears to be safe and may not portend an increased risk of recurrence. ${ }^{11-13}$

The average EBL was $228 \mathrm{ml}$, while average fluid resuscitation and urine output were $2336 \mathrm{ml}$ and $663 \mathrm{ml}$, respectively. Taken together, these patients usually do not require any blood transfusions and usually do not require significant amounts of crystalloid administration. This is beneficial, as excessive fluid resuscitation has been associated with postoperative complications, inclusive of pulmonary and tissue edema (which may affect wound healing), as well as hyperchloremic metabolic acidosis with subsequent acute renal injury. ${ }^{14}$ Blood transfusions have also been associated with risks, including immunological reactions of varying severity, laboratory errors leading to mismatch, the small risk of transfusion-associated infection, and similar sequelae of fluid overload with excessive administration..$^{15}$

The average LOS in this study was 4 days; 29 patients $(63 \%)$ were discharged to home, while 17 (37\%) were discharged to a rehabilitation facility. Our analysis did not reveal any significant factors predicting discharge to a facility, although age was trending toward significance. Older age would be an unsurprising risk factor for this outcome in spinal meningiomas, as increasing age has been associated with increased risk of preoperative neurological deficits and postoperative nonneurological complications. ${ }^{10}$ It is possible that a larger sample size would be more revealing for predictive factors in this cohort.

\section{Long-Term Postoperative Outcomes}

The median follow-up for this study was 53 months (range 1-201 months). Thirty patients had improved or resolved symptoms, 9 remained stable, and 6 worsened. Five patients died during follow-up, although none of these deaths occurred within 30 days of surgery. Only 1 death was related to the meningioma. This patient was a 50-year-old man with a WHO grade III anaplastic meningioma. The lesion was treated with STR, and the hospital course was complicated by postoperative hematomyelia and hemorrhagic infarct requiring reoperation. The patient received adjuvant radiotherapy with 31.8 Gy in 13 fractions. Despite this, the lesion progressed 1 month after surgery, and the patient died after receiving additional radiotherapy and sunitinib.

Only 1 patient, who was 70 years old at the time of surgery, required any subsequent spine surgery. Verte-

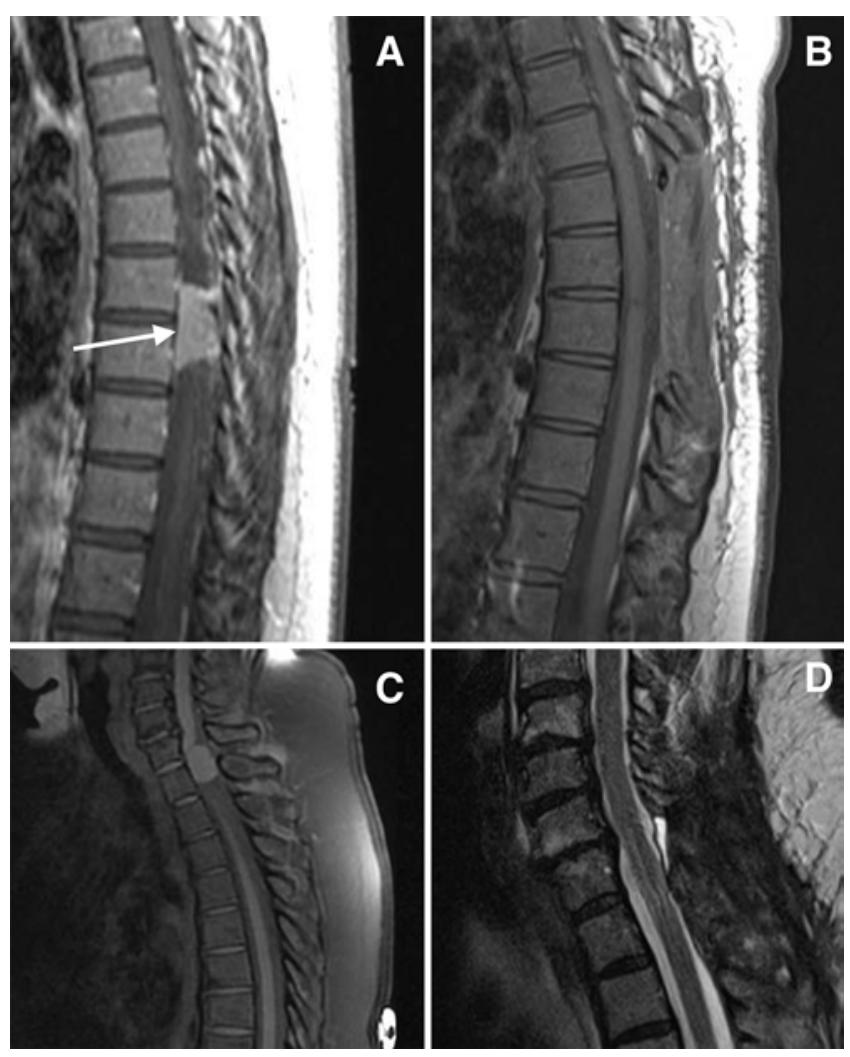

FIG. 1. Examples of ventral and dorsal spinal meningiomas. Preoperative T1-weighted contrast (A) and postoperative T1-weighted (B) MR images for a T8-9 ventral meningioma. Note the broad dural attachment located in the ventral part of the spinal canal (white arrow). Preoperative T1-weighted (C) and postoperative T2-weighted (D) MR images for a dorsal T1 meningioma.

broplasty was performed for compression fractures and resulting kyphoscoliosis near the level of the previously resected meningioma 8 years after the initial surgery. No additional patients required fusion or instrumentation.

\section{Study Limitations}

This study is limited by limitations inherent to all retrospective reviews. The sample size, although reasonable for a less common pathology like spinal meningiomas, was still relatively small for statistical purposes. Our analysis was limited to operative notes, and we cannot be confident about how wide or aggressive the bony decompressions differed between lead surgeons.

\section{Conclusions}

Surgery for thoracic spinal meningiomas is well tolerated and safe. The ability of a surgeon to obtain adequate resection was not limited by a ventral position of the neoplasm. Overall, patients did not require blood transfusion or excessive intraoperative fluid resuscitation. Only 1 patient required concurrent instrumentation and fusion at the time of resection. Most patients were discharged to home, and increasing age was trending toward significance for those patients discharged to rehabilitation facilities. 


\section{References}

1. Engelhard HH, Villano JL, Porter KR, et al. Clinical presentation, histology, and treatment in 430 patients with primary tumors of the spinal cord, spinal meninges, or cauda equina. J Neurosurg Spine. 2010;13(1):67-77.

2. Yamaguchi S, Menezes AH, Shimizu K, et al. Differences and characteristics of symptoms by tumor location, size, and degree of spinal cord compression: a retrospective study on 53 surgically treated, symptomatic spinal meningiomas. $J$ Neurosurg Spine. 2020;32(6):931-940.

3. Beall DP, Googe DJ, Emery RL, et al. Extramedullary intradural spinal tumors: a pictorial review. Curr Probl Diagn Radiol. 2007;36(5):185-198.

4. Gezen F, Kahraman S, Çanakci Z, Bedük A. Review of 36 cases of spinal cord meningioma. Spine (Phila Pa 1976). 2000;25(6):727-731.

5. Gilard V, Goia A, Ferracci FX, et al. Spinal meningioma and factors predictive of post-operative deterioration. J Neurooncol. 2018;140(1):49-54.

6. Levy WJ Jr, Bay J, Dohn D. Spinal cord meningioma. J Neurosurg. 1982;57(6):804-812.

7. Solero CL, Fornari M, Giombini S, et al. Spinal meningiomas: review of 174 operated cases. Neurosurgery. 1989;25(2): $153-160$

8. Maiti TK, Bir SC, Patra DP, et al. Spinal meningiomas: clinicoradiological factors predicting recurrence and functional outcome. Neurosurg Focus. 2016;41(2):E6.

9. Sacko O, Haegelen C, Mendes V, et al. Spinal meningioma surgery in elderly patients with paraplegia or severe paraparesis: a multicenter study. Neurosurgery. 2009;64(3):503-510.

10. Schwake M, Adeli A, Sporns P, et al. Spinal meningiomasrisks and potential of an increasing age at the time of surgery. J Clin Neurosci. 2018;57:86-92.

11. Ito K, Aoyama T, Miyaoka Y, et al. Surgery for ventral intradural thoracic spinal tumors with a posterolateral transpedicular approach. Acta Neurochir (Wien). 2016;158(8): $1563-1569$.

12. Takami T, Naito K, Yamagata T, et al. Posterolateral approach for spinal intradural meningioma with ventral attachment. J Craniovertebr Junction Spine. 2015;6(4):173-178.
13. Tola S, De Angelis M, Bistazzoni S, et al. Hemilaminectomy for spinal meningioma: a case series of 20 patients with a focus on ventral- and ventrolateral lesions. Clin Neurol Neurosurg. 2016;148:35-41.

14. Myles PS, Andrews S, Nicholson J, et al. Contemporary approaches to perioperative IV fluid therapy. World J Surg. 2017;41(10):2457-2463.

15. Ferraris VA, Ferraris SP, Saha SP, et al. Perioperative blood transfusion and blood conservation in cardiac surgery: the Society of Thoracic Surgeons and the Society of Cardiovascular Anesthesiologists clinical practice guideline. Ann Thorac Surg. 2007;83(5)(suppl):S27-S86.

\section{Disclosures}

Dr. C. Shaffrey: consultant for Medtronic, NuVasive, and SI Bone; direct stock ownership in NuVasive; royalties from Medtronic and NuVasive; and patent holder with Medtronic, NuVasive, and Zimmer Biomet. Dr. Smith: consultant for Zimmer Biomet, NuVasive, Cerapedics, Carlsmed, Stryker, and DePuy Synthes; direct stock ownership in Alphatec and NuVasive; and fellowship support from AO Spine.

\section{Author Contributions}

Conception and design: Ampie. Acquisition of data: Ampie, Snyder. Analysis and interpretation of data: Ampie, Snyder. Drafting the article: Ampie, Snyder. Critically revising the article: all authors. Reviewed submitted version of manuscript: all authors. Approved the final version of the manuscript on behalf of all authors: Ampie. Statistical analysis: Snyder. Study supervision: Smith.

\section{Supplemental Information Current Affiliations \\ Dr. Syed: Children's National Hospital, Washington, DC.}

\section{Correspondence}

Leonel Ampie: University of Virginia Health System, Charlottesville, VA. leonel.ampie@nih.gov. 